Research Article

\title{
Antioxidant Activity In Vitro Guided Screening and Identification of Flavonoids Antioxidants in the Extract from Tetrastigma hemsleyanum Diels et Gilg
}

\author{
Li Ding $(\mathbb{D}$, Xiaomin Zhang, and Jiajia Zhang \\ Zhejiang Pharmaceutical College, Ningbo, Zhejiang, China \\ Correspondence should be addressed to Li Ding; nbncdl@163.com
}

Received 17 July 2021; Accepted 2 November 2021; Published 23 November 2021

Academic Editor: Valentina Venuti

Copyright (C) 2021 Li Ding et al. This is an open access article distributed under the Creative Commons Attribution License, which permits unrestricted use, distribution, and reproduction in any medium, provided the original work is properly cited.

\begin{abstract}
This study aimed to investigate the extract with high antioxidant activity of Tetrastigma hemsleyanum Diels et Gilg and identify the antioxidant components in vitro. $\alpha, \alpha$-Diphenyl- $\beta$-picrylhydrazyl (DPPH) radical assay, Trolox equivalent antioxidant capacity (TEAC) assay, ferric reducing antioxidant power (FRAP), and hydroxyl radical scavenging method were used to screen the extract with high antioxidant activity. The antioxidant capacity of the extracts was evaluated by the free radical scavenging ability of $\mathrm{DPPH}$. The ability of extracts to scavenge 2, 2'-azino-bis(3-ethylbenzothiazoline-6-sulfonic acid) (ABTS) free radical was evaluated by TEAC assay. The FRAP method was used to evaluate the ability of extracts to reduce $\mathrm{Fe}^{3+}$. The ability to scavenge hydroxyl radicals produced by the interaction of hydrogen peroxide and $\mathrm{Fe}^{2+}$ was measured by monitoring the change in the absorbance of the reaction mixture at $536 \mathrm{~nm}$. Then, high-performance liquid chromatography-DPPH (HPLC-DPPH) and HPLC-hydroxyl radical scavenging methods were used to screen the antioxidant components in the extract. The molecular weight of the above antioxidant components was investigated using the qualitative analytical method of high-performance liquid chromatography coupled with quadrupole time-of-flight tandem mass spectrometry (HPLC-Q-TOF LC/MS). Based on the concentrations of the samples $(0.2-4 \mathrm{mg} / \mathrm{mL})$, the DPPH free radical scavenging ability, ABTS+ free radical scavenging ability, hydroxyl free radical scavenging ability, and $\mathrm{Fe}^{3+}$ reducing ability of the ethyl acetate extract (EAE) were stronger than that of the crude extract (CE), petroleum ether extract (PEE), and n-butanol extract (BE). The EAE has higher antioxidant activity than CE, $\mathrm{PEE}$, and BE. Six antioxidant components, rutin, quercetin, isoquercetin, astragalin, kaempferol, and kaempferol-3-o-rutoside, were identified in the EAE.
\end{abstract}

\section{Introduction}

The rhizome of Tetrastigma hemsleyanum Diels et Gilg, a Chinese folk herbal medicine, is used for the treatment of children with high fever, pneumonia, hepatitis, viral meningitis, scrofula, carbuncle, and furuncle [1]. Modern pharmacological studies have shown that Tetrastigma hemsleyanum Diels et Gilg has anti-inflammatory, analgesic [2], antiviral [3], antiliver injury [4, 5], antitumor [6,7], and other pharmacological effects $[8,9]$.

A large number of studies are focused on the chemical constituents of Tetrastigma hemsleyanum Diels et Gilg. The aboveground part of Tetrastigma hemsleyanum Diels et Gilg contains $\beta$-sitosterol, palmitic acid, pentacosane, gallic acid, ethyl gallate, catechin, 7-O-galloylcatechin, and 3,3'- dimethoxy ellagic acid-4-O- $\beta$-D-glucopyranoside [10]. Taraxerone, ergosterol, oleic acid, palmitic acid, myristic acid, hexadecylic acid, heptadecanoic acid, 9,12-octadecadienoic acid, $\alpha$-linolenic acid, kaempferol, quercetin, and kaempferol-3o-neohesperidin were found in liposolubility extract of Tetrastigma hemsleyanum Diels et Gilg [11-13]. In addition, $\mathrm{Xu}$ analyzed $80 \%$ methanol ultrasonic extract of Tetrastigma hemsleyanum by HPLC-Q-TOF-MS and found that it contained kaempferol-7-o-rhamnose-3-o-glucoside, rutin, isoquercetin, kaempferol rutoside, astragaloside, and quercetin [12]. Fan identified 8 flavonoids (isoorientin, orientin, orientin-2"-O-rhamnoside, isoorientin-2"-O-rhamnoside, vitexin, vitexin-2"-O-rhamnoside, isovitexin, and 
isovitexin-2"-O-rhamnoside) in Tetrastigma hemsleyanum leaves by rapid resolution liquid chromatography coupled with quadrupole time-of-flight tandem mass spectrometry (RRLC-Q-TOF-MS) [14]. These results showed that oleic acid and flavonoids constituted the largest proportion of the plant [15]. Flavonoids have various biological activities, such as the cardiovascular system $[16,17]$, antioxidant $[18-20]$, anti-tumor [21-23], anti-inflammatory, and analgesic [24], and are one of the most and diverse phenolic groups of natural origin [25]; their antioxidation capability is a significant property.

Free radicals that are produced in the process of life metabolism regulate the signal transmission between cells and cell growth and inhibit the viruses and bacteria [26]. Excessive free radicals attack the cell membrane and leak from the intracellular region, which in turn leads to the denaturation of protein and nucleic acid, thereby, damaging the cells, tissues, and organs, inducing various diseases, and accelerating the aging of the body. The mechanism of inflammation, tumor, aging, and cardiovascular diseases is closely related to the excessive production of free radicals or a decrease in the ability to scavenge free radicals [27]. Antioxidants quench free radicals and form nontoxic ions or molecules. The free radicals were scavenged to achieve antioxidation, and antioxidants can stop or reverse some damage caused by excessive free radicals [28]. Synthetic antioxidants used to prolong the shelf life of food, such as butyl hydroxyanisole (BHA), dibutyl hydroxytoluene (BHT), and propyl gallate (PG) [29-31], have been found to be potential hepatotoxicity and carcinogenicity $[32,33]$. Due to restricted use of synthetic antioxidants [34], natural antioxidants with high safety and fewer side effects are favored. Natural antioxidants and active substances mainly originate from plants [31], including tea extract $[35,36]$, fruit and vegetable extracts [37], balsam pear extracts [38], Chinese herbal extracts [39], raw Rehmanniae extract [40], and spices extracts [41]. Natural antioxidants can eliminate free radicals $[42,43]$, improve blood circulation, delay cell aging, and prevent cancer $[44,45]$.

While screening the antitumor extract and active components of Tetrastigma hemsleyanum Diels et Gilg, we found that some of the isolated parts were oxidized $[46,47]$. This phenomenon indicated that there might be antioxidant components in Tetrastigma hemsleyanum Diels et Gilg. However, active components responsible for antioxidants in Tetrastigma hemsleyanum Diels et Gilg are yet to be identified. Therefore, by utilizing the antioxidant test, we screened out the extract that harbors high antioxidant activity, and the molecular weight of the potential active components was determined by HPLC-Q-TOF LC/MS.

\section{Materials and Methods}

2.1. Chemicals and Plant Material. DPPH, ABTS, TPTZ (2,4,6-tri-2-pyridinyl-1,3,5-triazine), and Trolox (6-hydroxy-2,5,7,8-tetramethylchroman-2-carboxylic acid) were purchased from Sigma (St. Louis, MO, USA), and methanol, ethanol, petroleum ether, ethyl acetate, n-butanol, and other reagents for the antioxidant test were analytically pure and purchased from China National Pharmaceutical Group Co. (Beijing, China). Rutin (lot no. 100080-201805, HPLC $\geq 98 \%$ ), astragaloside (lot no. 110781-201908, HPLC $\geq 98 \%$ ), isoquercetin (lot no. 111809-201804, HPLC $\geq 98 \%$ ), quercetin (lot no. 100081-201610, HPLC $\geq 98 \%$ ), kaempferol (lot no. 110861-201703, HPLC $\geq 98 \%$ ), and kaempferol-3-o-rutoside (lot no. 112007-201903, HPLC $\geq 98 \%$ ) standard reference materials were obtained from National Institutes for Food and Drug Control (Beijing, China). Chromatography grade and LC-MS grade acetonitrile were obtained from Tedia (Fairfield, OH, USA) and Fisher (Waltham, MA, USA), respectively.

The rhizome of Tetrastigma hemsleyanum Diels et Gilg from Guangxi Province, China, was purchased from Guangxi, China (lot no. 180615), and identified by Professor Xia Miao-fen at Zhejiang Pharmaceutical College.

\subsection{Extraction, Fractionation, and Antioxidant Evaluation.}

The extracts were prepared according to the method of Bao et al. [48] and Yong [49] with minor modifications. The dried powder $(5 \mathrm{~kg})$ of the rhizomes of Tetrastigma hemsleyanum Diels et Gilg was soaked in three volumes of $60 \%$ methanol at room temperature. Subsequently, the soaking solution was filtered, combined, and concentrated under reduced pressure; the crude extract (CE) (364g) was prepared by vacuum drying. Then, it was suspended in water and extracted successively with petroleum ether, ethyl acetate, and n-butanol to obtain petroleum ether extract (PEE) (28g), ethyl acetate extract (EAE) (25g), and n-butanol extract (BE) (68g), respectively. The antioxidant activities of each extract were measured using the DPPH assay, TEAC assay, ferric reducing antioxidant power (FRAP), and hydroxyl radical scavenging method to screen the extract with high antioxidant activity. The antioxidant components in this extract were detected by HPLC-DPPH and HPLC-hydroxyl radical scavenging methods. The molecular weight of the antioxidant components was deduced by HPLC-Q-TOF LC/MS. The extracts were solubilized in ethanol solution $(80 \% \mathrm{v} / \mathrm{v})$, except for PEE, which was dissolved in dimethyl sulfoxide (DMSO).

2.3. DPPH Assay. The DPPH free radical scavenging activity was assessed according to Leong et al. [50] with a slight improvement. Briefly, $3.0 \mathrm{~mL}$ of DPPH $80 \%$ ethanol solution $\left(10^{-4} \mathrm{M}\right)$ was mixed with $0.1 \mathrm{~mL}$ of the sample solution. After incubation for $30 \mathrm{~min}$ in the dark at room temperature, the absorbance was measured at $517 \mathrm{~nm}$ using a UV-vis spectrophotometer (Shimadzu UV-1800, Kyoto, Japan). The radical scavenging activity (\%) was calculated as follows:

$$
\text { Scavenging activity }(\%)=\left[\frac{1-A_{1}}{A}\right] \times 100 \%,
$$

where $A_{1}$ is the absorbance of the sample, and $A_{0}$ is the absorbance of the blank group.

2.4. TEAC Assay. The antioxidant activity against ABTS+ was measured as described by Strail et al. [51] with some modifications. ABTS+ was formed by reacting $7.0 \mathrm{mM}$ ABTS with $4.95 \mathrm{mM}$ potassium persulfate solution at room 
temperature in the dark for $12 \mathrm{~h}$. Then, the solution was diluted with $80 \%$ ethanol before measuring the absorbance $(0.70 \pm 0.02)$ at $734 \mathrm{~nm}$. After incubation of $20 \mu \mathrm{L}$ sample solution $(0.2-4 \mathrm{mg} / \mathrm{mL})$ with $3.0 \mathrm{~mL}$ ABTS+ at $30^{\circ} \mathrm{C}$ for $10 \mathrm{~min}$, the absorbance was measured at $734 \mathrm{~nm}$ by the UVvis spectrophotometer. The antioxidant capacity was expressed as $\mathrm{mg}$ of Trolox/g of samples (TEAC), and the TEAC was calculated as follows:

$$
\text { TEAC }=\frac{\left(A_{0}-A_{1}\right)-a}{b} \times \frac{V}{W},
$$

where $A_{1}$ is the absorbance of the sample, $A_{0}$ is the absorbance of the blank group, $b$ is the slope of the standard curve, $a$ is the intercept of the standard curve, $V$ is the volume of Trolox $(\mu \mathrm{L})$, and $W$ is the weight of extracts $(\mathrm{g})$.

2.5. FRAP. The antioxidant activity against $\mathrm{Fe}^{3+}-\mathrm{TPTZ}$ was performed according to the method established by Benzie et al. [52] with some modifications. Fresh FRAP reagent was prepared by mixing $300 \mathrm{~mm}$ acetate buffer ( $\mathrm{pH} 3.6$ ), $10 \mathrm{~mm}$ TPTZ solution, and $20 \mathrm{~mm} \mathrm{FeCl} \cdot 6 \mathrm{H}_{2} \mathrm{O}$ solution at the ratio of $10: 1: 1$. A volume of $3.0 \mathrm{~mL}$ FRAP reagent was added to $20 \mathrm{~L}$ sample solution $(0.2-4 \mathrm{mg} / \mathrm{mL})$ and incubated at $37^{\circ} \mathrm{C}$ for $30 \mathrm{~min}$. The absorbance of the reaction mixture was measured at $593 \mathrm{~nm}$. FRAP was expressed as the number of moles of $\mathrm{FeSO}_{4}$ required to achieve the same absorbance per $\mathrm{g}$ of samples and calculated as follows:

$$
\text { FRAP }=\frac{\left[A_{1}-A_{0}\right]-a}{b} \times \frac{1}{W},
$$

where $A_{1}$ is the absorbance of the sample, $A_{0}$ is the absorbance of the blank group, $b$ is the slope of the standard curve, $a$ is the intercept of the standard curve, and $W$ is the weight of extracts $(\mathrm{g})$.

2.6. Hydroxyl Radical Scavenging Assay. The hydroxyl radical scavenging capacity was evaluated according to the method described by Jin et al. [53] with some modifications. Briefly, $1.0 \mathrm{~mL}$ phosphate-buffered saline (PBS, pH 7.4) was mixed with $0.5 \mathrm{~mL}(0.148 \mathrm{mg} / \mathrm{mL})$ o-phenanthrene solution, $0.5 \mathrm{~mL}(0.208 \mathrm{mg} / \mathrm{mL})$ ferrous sulfate solution, and $1.0 \mathrm{~mL}$ sample solution $(0.2-4 \mathrm{mg} / \mathrm{mL})$. After the mixture was vortexed, $1.0 \mathrm{~mL}(0.01 \%)$ hydrogen peroxide $\left(\mathrm{H}_{2} \mathrm{O}_{2}\right)$ solution was added and incubated at $37^{\circ} \mathrm{C}$ for $60 \mathrm{~min}$. The absorbance was measured at $536 \mathrm{~nm}$. The hydroxyl radical scavenging capacity was calculated as follows:

$$
\text { Scavenging activity }(\%)=\left[\frac{\left(A_{1}-A_{0}\right)}{\left(A_{2}-A_{0}\right)}\right] \times 100 \%,
$$

where $A_{1}$ is the absorbance of the sample, $A_{0}$ is the absorbance of the blank control group, and $A_{2}$ is the absorbance of the sample and reagent blank group.

2.7. HPLC Conditions. Herein, we used traditional methods to select the extract with high antioxidant activity, and the antioxidant components were screened using the HPLC-
DPPH scavenging method and HPLC-hydroxyl radical scavenging method [54]. The chromatography selected optimal chromatographic conditions to separate the components in the extract. Then, the content of the sample components before and after the addition of the free radicals was detected using a UV detector. Next, the components with antioxidant capacity were selected by comparing the changes in the peak area or peak height of the corresponding components in the two chromatograms. Antioxidant components in the extract with high oxidation activity were screened by using an Agilent HPLC system with a photodiode-array detector (1260 series, Santa Clara, CA, USA). The chromatographic separation was carried out using a Shimadzu VP-ODS C18 $(150 \times 4.6 \mathrm{~mm}, 5 \mu \mathrm{m})$ with solvent A ( $0.1 \%$ formic acid) and solvent B (acetonitrile). The gradient condition was as follows: $0 \mathrm{~min}, 18 \% \mathrm{~B} ; 0-5 \mathrm{~min}, 25 \% \mathrm{~B}$; 5-10 min, 35\% B; 10-15 min, 60\% B; 15-20 min, 90\% B; and 20-30 min, $18 \% \mathrm{~B}$. The flow rate was $1.0 \mathrm{~mL} / \mathrm{min}$, the injection volume was $20 \mu \mathrm{L}$, the column temperature was set at $30^{\circ} \mathrm{C}$, and the detection wavelength was $254 \mathrm{~nm}$.

2.8. HPLC-DPPH Scavenging Method. The majority of the phenolic antioxidants can quench DPPH radicals, and hence, DPPH radicals are often used to screen the antioxidant activities of phenolic compounds [55]. In this study, we used the HPLC-DPPH scavenging method to determine the peak area of each component in the sample before and after adding DPPH. The components of the reduced peak area harbored antioxidant activity. Briefly, $250 \mu \mathrm{L}$ sample solution $(4 \mathrm{mg} / \mathrm{L})$ and $250 \mu \mathrm{L} \mathrm{DPPH}$ solution $(500 \mu \mathrm{M})$ were mixed and incubated at $37^{\circ} \mathrm{C}$ in the dark for $30 \mathrm{~min}$. The mixture was then filtered through a $0.45 \mu \mathrm{m}$ membrane and determined according to the chromatographic conditions in 2.2.5.

2.9. HPLC-Hydroxyl Radical Scavenging Method. Briefly, $200 \mu \mathrm{L} \mathrm{H}_{2} \mathrm{O}_{2}$ solution ( $25 \mathrm{mM}$ ) and $200 \mu \mathrm{L}$ of $\mathrm{FeSO}_{4}$ solution $(250 \mu \mathrm{M})$ were added to $250 \mu \mathrm{L}$ sample solution $(4 \mathrm{mg} / \mathrm{L})$. The mixture was incubated at room temperature in the dark for $7 \mathrm{~min}$, filtered with $0.45 \mu \mathrm{m}$ membrane, and measured according to the chromatographic conditions in 2.2.5.

2.10. Identification of Active Antioxidant Ingredients. The identification experiment was carried out using an Agilent HPLC-Q-TOF LC/MS (1260 series, 6520 series) and an Agilent HPLC system with a photodiode array detector (1260 series). The chromatographic separation was conducted using a Extend Agilent C18 $(50 \times 2.1 \mathrm{~mm}, 1.8 \mu \mathrm{m})$ with solvent A ( $0.1 \%$ formic acid) and solvent B (acetonitrile). The gradient condition was as follows: $0 \mathrm{~min}, 18 \% \mathrm{~B}$; 0-5 min, $25 \% \mathrm{~B}$; $5-10 \mathrm{~min}, 35 \% \mathrm{~B} ; 10-15 \mathrm{~min}, 60 \% \mathrm{~B}$; and $15-20 \mathrm{~min}, 90 \% \mathrm{~B}$. The flow rate was $0.2 \mathrm{~mL} / \mathrm{min}$, injection volume was $1 \mu \mathrm{L}$, the column temperature was room temperature, the scanning range was $50-1500$, and the capillary voltage was $4000 \mathrm{~V}$. Nitrogen was used as dry gas at a flow rate of $10 \mathrm{~mL} / \mathrm{min}$, the temperature was $350^{\circ} \mathrm{C}$, and the atomizer pressure was $40 \mathrm{psi}$. The mass spectrogram and 
chromatogram were recorded under the negative ion source. After acquiring the molecular weight, the antioxidant components in the sample were further identified based on their standard products by HPLC, according to the chromatographic conditions in 2.2.5.

2.11. Statistical Analysis. All experimental data were expressed as mean \pm standard deviation (SD) of three independent experiments in triplicate and analyzed by SPSS (version 17.0). Oneway analysis of variance (ANOVA) and Tukey multiple comparisons were carried out to test any significant differences between the means. $P<0.05$ indicated statistical significance.

\section{Results}

\subsection{Screening of Antioxidant Extracts}

3.1.1. Comparison of DPPH Free Radical Scavenging Capacity of Extracts. DPPH has been widely used in the determination of antioxidant free radical scavenging capacity [56]. The scavenging rate of each extract is shown in Figure 1. Subsequently, the DPPH scavenging activity of EAE and CE was similar to the sample concentrations of $0.2 \mathrm{mg} / \mathrm{mL}(P>0.05)$ and $4 \mathrm{mg} / \mathrm{mL}(P>0.05)$, respectively, while DPPH scavenging activity of EAE and all the other extracts is the largest difference at the sample concentration of $1 \mathrm{mg} / \mathrm{mL}(P<0.05)$. The DPPH scavenging activity of EAE was dose-dependent in the concentration range of $0.2-4 \mathrm{mg} / \mathrm{mL}$ and occurred in the following order: $\mathrm{EAE}>\mathrm{CE}>\mathrm{BE}>\mathrm{PEE}$ (Figure 2).

3.1.2. Comparison of ABTS+ Free Radical Scavenging Capacity of Extracts. The TEAC values of different concentrations of each extract are shown in Figure 2. In addition to PPE, the other extracts showed a robust ability to scavenge ABTS+ radicals. However, no significant difference was detected in the TEAC values between EAE and $\mathrm{CE}$ at the sample concentrations of $0.2,0.5$, and $1 \mathrm{mg} / \mathrm{mL}(P>0.05)$. On the other hand, in the sample concentration range of $0.2-4 \mathrm{mg} / \mathrm{mL}$, the TEAC values of EAE were $150.78 \pm 20.94,241.72 \pm 30.34$, $281.36 \pm 3.17,265.54 \pm 7.79$, and $207.59 \pm 14.45 \mathrm{mg}$ Trolox/g extract, respectively. The TEAC value was not dose-dependent because the weight of the extract $(W)$ varied in the five different concentrations of each extract. If calculated according to formula (1), the antioxidant capacity was dose-dependent and expressed in terms of scavenging activity.

3.1.3. Comparison of the Reducing Power of Extracts. In this experiment, the reduction ability of CE and EAE was dissimilar $(P<0.05)$. At different concentrations, the reduced ability of EAE was significantly higher than that of other extracts, indicating a high reduction ability of $\mathrm{Fe}^{3+}$. In the concentration range of $0.2-4 \mathrm{mg} / \mathrm{mL}$, the FRAP values of EAE were $0.74 \pm 0.06,1.12 \pm 0.04,1.02 \pm 0.04,0.71 \pm 0.01$, and $0.68 \pm 0.00 \mathrm{mM} \mathrm{FeSO}_{4} / \mathrm{g}$ extract, respectively (Figure 3).

3.1.4. Comparison of Hydroxyl Radicals Scavenging Capacity of Extracts. Figure 4 shows the highest clearance rate of EAE, although the clearance rates of CE of 1 and $2 \mathrm{mg} / \mathrm{mL}$

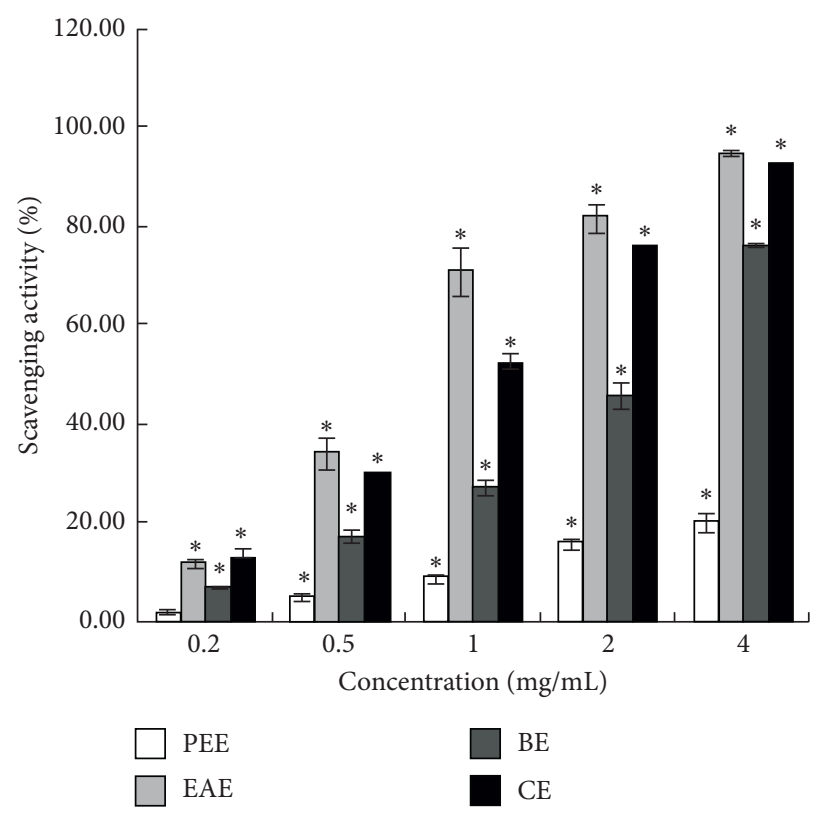

FIGURE 1: DPPH radical scavenging activity (\%) of four extracts. ${ }^{*} P<0.05$ vs. the blank group.

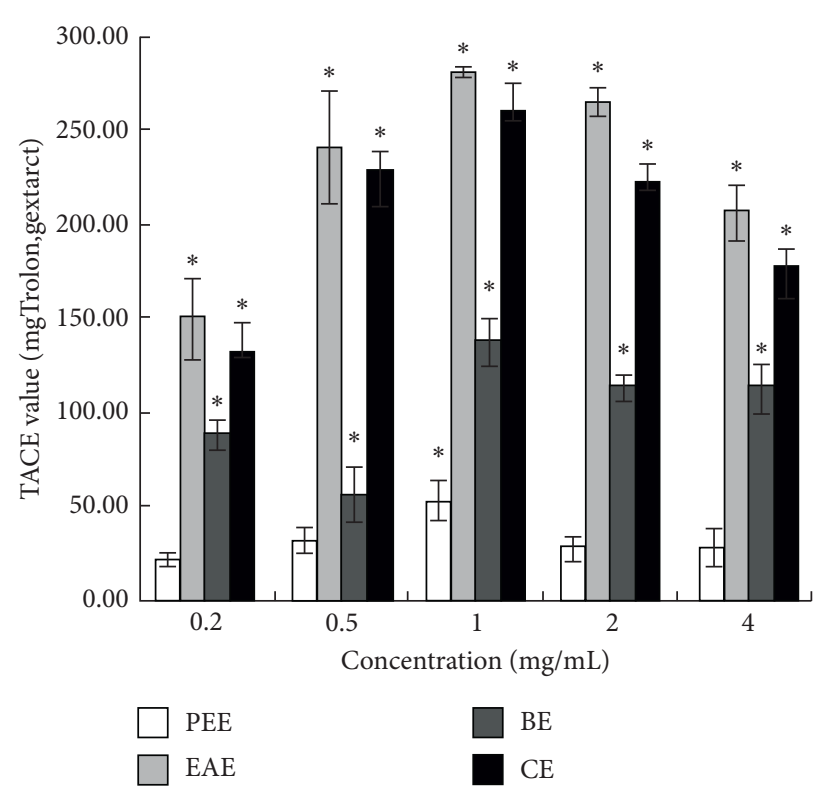

Figure 2: TEAC value (Trolox mg/g extract) of four extracts. ${ }^{*} P<0.05$ vs. the blank group.

concentrations are close to that of $\operatorname{EAE}(P>0.05)$. At $0.2,0.5$, and $0.4 \mathrm{mg} / \mathrm{mL}$, the clearance rate of EAE was significantly different from that of other extracts $(P<0.05)$. In the concentration range of $0.2-4 \mathrm{mg} / \mathrm{mL}$, the scavenging activity of hydroxyl radicals in EAE was $32.69 \pm 3.66 \%$, $59.73 \pm 2.76 \%, \quad 56.80 \pm 1.69 \%, \quad 51.28 \pm 3.47 \%$, and $47.60 \pm 1.63 \%$, respectively.

3.2. Screening Antioxidant Components in the Extract with High Antioxidant Activity by HPLC. The present study demonstrated that the EAE had the strongest antioxidant 


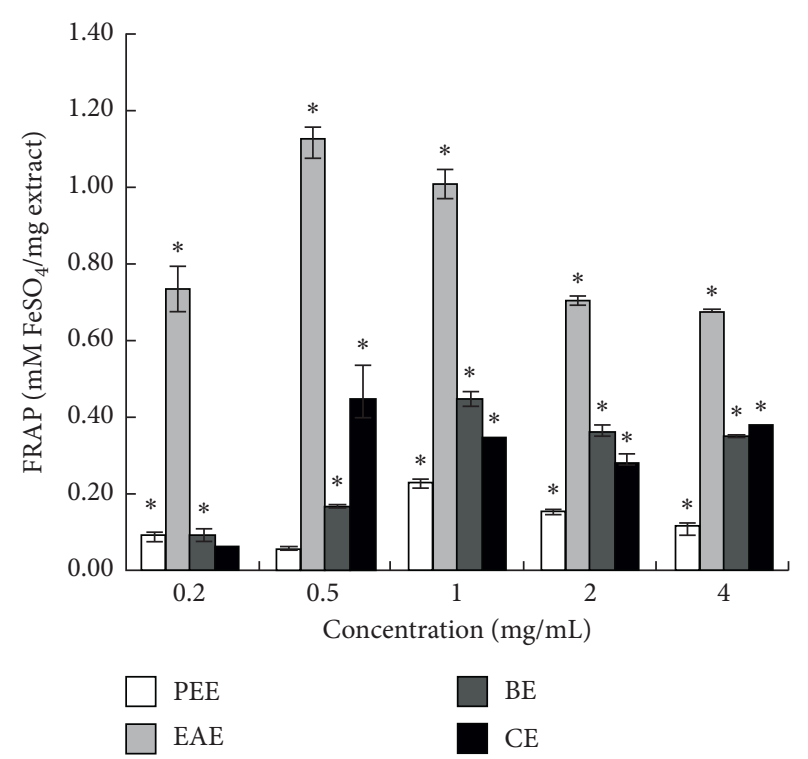

FIgURE 3: FRAP value $\left(\mathrm{FeSO}_{4} \mathrm{mM} / \mathrm{mg}\right.$ extract) of four extracts. ${ }^{*} P<0.05$ vs. the blank group.

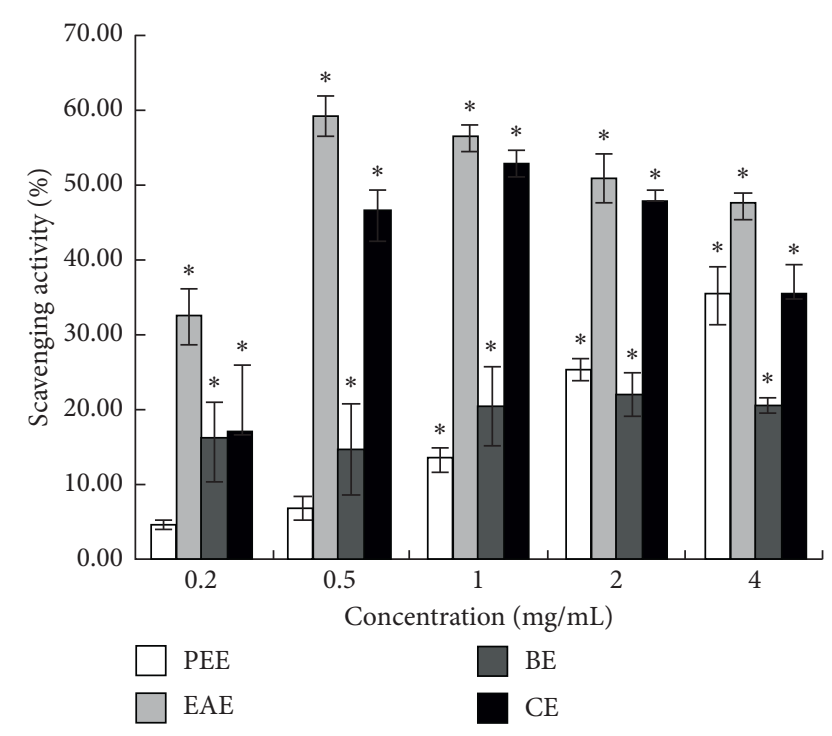

FIgURE 4: Hydroxyl radicals scavenging activity (\%) of four extracts. ${ }^{*} P<0.05$ vs. the blank group.

activity among the four extracts. Then, we used HPLC to analyze and screen the antioxidant components by HPLC-DPPH and HPLC-hydroxyl radical scavenging methods.

When no reagent was added to the sample solution, nine chromatographic peaks were obtained (Figure 5(a)). After the DPPH solution (Figure 5(b)) or hydroxyl radical reagent (Figure 5(c)) was added to the sample solution, distinct changes were detected in the area and height of the nine peaks. Compared to the chromatograms of the sample before and after the reaction, the amount of free radicals and the antioxidant content decreased with the progress in the antioxidant reaction (Figure 5). A total of nine components were identified as peaks.
3.3. Identifying Antioxidant Components in the Extract with High Antioxidant Activity. The response of each component in the EAE extract to the negative ion source is better than that of the positive ion source, which is the same as reported previously $[57,58]$. Based on the total ion chromatogram (Figure 6) and six-component mass spectrogram (Figure 7) under the negative ion detection mode and the molecular weight and molecular structure (Table 1) analyzed by the data of the workstation, we speculated the potential compounds and compared them with the corresponding standards by HPLC. Finally, we identified six compounds, namely, rutin, isoquercetin, astragaloside, quercetin, kaempferol, and kaempferol-3-o-rutoside (Figure 8).

\section{Discussion}

The current results are different from those of Sun et al. that $80 \%$ methanol extract of Tetrastigma hemsleyanum had better scavenging ability to DPPH and ABTS+ free radicals than ethyl acetate extract and hexane extract [49], which could be attributed to different extraction methods, origins of the herb, and concentrations of the samples. In this study, the total antioxidant capacity of $\mathrm{CE}$ was not equivalent to that of EAE, although the trend of CE in scavenging DPPH radicals, ABTS+ radicals, hydroxyl radicals, and reducing $\mathrm{Fe}^{3+}$ was consistent with that of EAE (Figures 1-4). This might be ascribed to the lower content of flavonoids in $\mathrm{CE}$ than that in EAE.

Figure 5 shows that the content of peaks 1,2, and 3 also decreased; however, the molecular weight was not determined by HPLC-Q-TOF LC/MS. Furthermore, phenolic acids, such as catechin, epicatechin, gallic acid, procyanidin $\mathrm{B}_{1}$, and procyanidin $\mathrm{B}_{2}[59,60]$, with strong antioxidant activity, have not been identified in EAE, CE, PEE, and BE, which might be due to the extraction process or the low content of phenolic acids in the extracts. The antioxidative activity of EAE in vivo and the distribution and metabolism of each antioxidant component in the organisms are not clear, and the mechanism of EAE underlying the antioxidative effect remains to be elucidated.

The six identified compounds were flavonoids and polyhydroxy compounds, and their antioxidant activity has been reported. Among these, rutin is a strong antioxidant [57], and its antioxidant activity is based on three mechanisms: the o-dihydroxy structure in $\mathrm{B}$ ring, the 2,3 double bond of the 4-oxo-functional group coupling in $\mathrm{C}$ ring, and the 5-OH and 7-OH groups of the 4-oxo-functional group in $\mathrm{A}$ and $\mathrm{C}$ rings. Rutin alleviates early brain injury of rats with subarachnoid hemorrhage by antioxidation and antiapoptosis [58]. Quercetin inhibits the activities of acetylcholinesterase and butyryl cholinesterase and $\mathrm{Fe}^{2+}$ induces lipid peroxidation in the brain homogenates of the rat, which are the putative mechanisms that underlie the quercetinmediated management of oxidative stress-induced neurodegeneration [61]. Isoquercetin, a potent antioxidative compound, has neuroprotective capacities that are beneficial for the treatment of ischemic stroke and related diseases [62]. Astragalin, a major flavonoid, is a potential beneficial agent that protects the diabetic-induced spermatogenic 


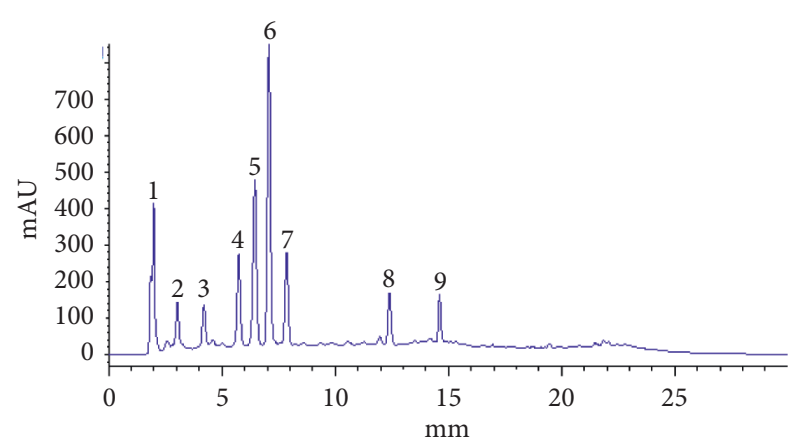

(a)

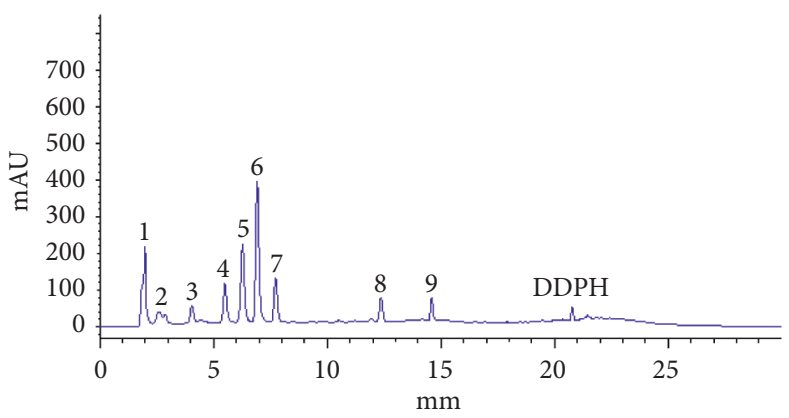

(b)

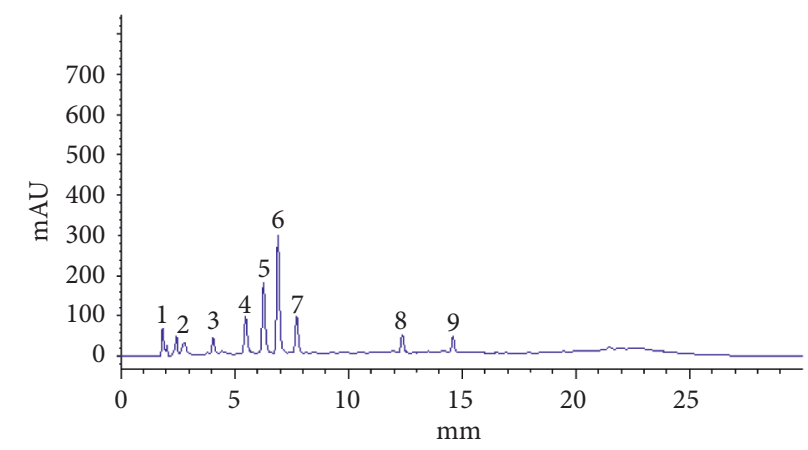

(c)

FIGURE 5: Chromatogram of antioxidant components in EAE (4 mg/mL) screened by HPLC. (a) Chromatogram of the sample without any added reagent. (b) Chromatogram of the sample with added DPPH. (c) Chromatogram of the sample with added hydroxyl radical.

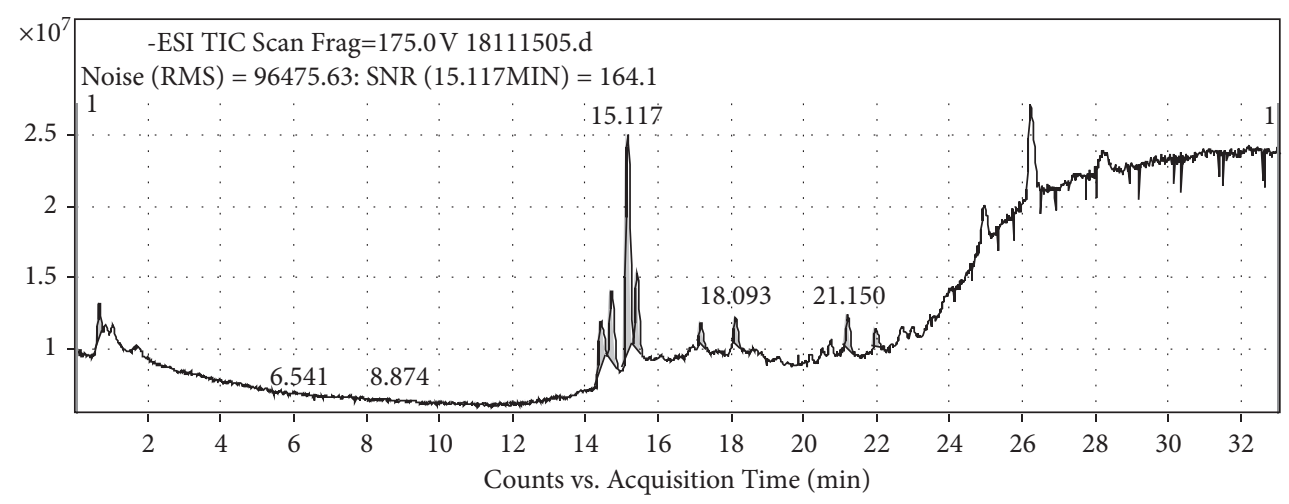

Figure 6: Total ion current diagram of EAE $(4 \mathrm{mg} / \mathrm{mL})$.

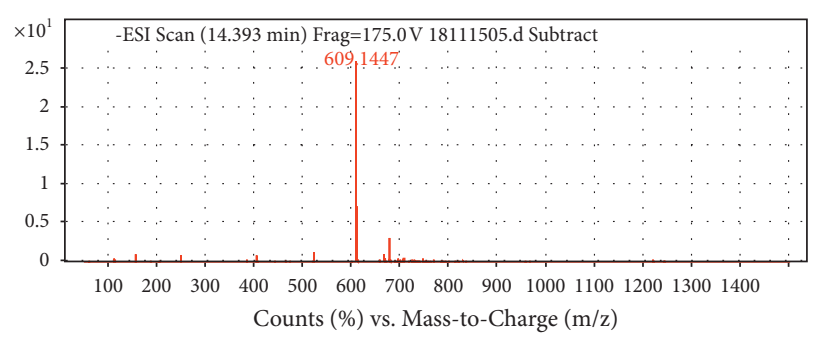

(a)

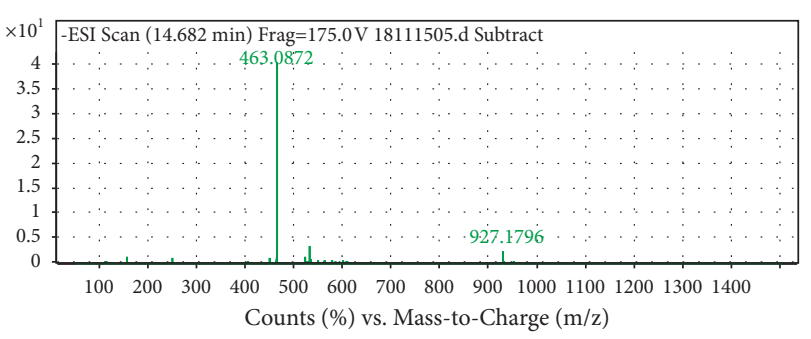

(b)

Figure 7: Continued. 


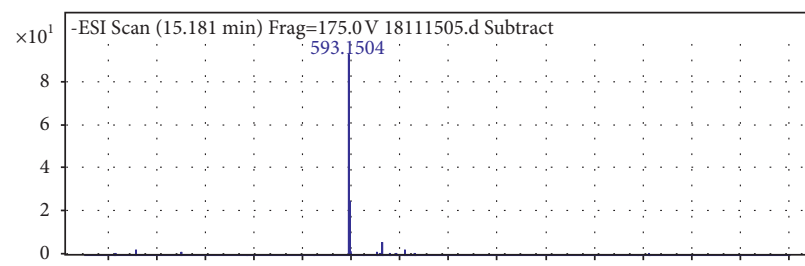

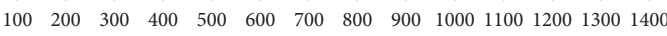
Counts (\%) vs. Mass-to-Charge $(\mathrm{m} / \mathrm{z})$

(c)

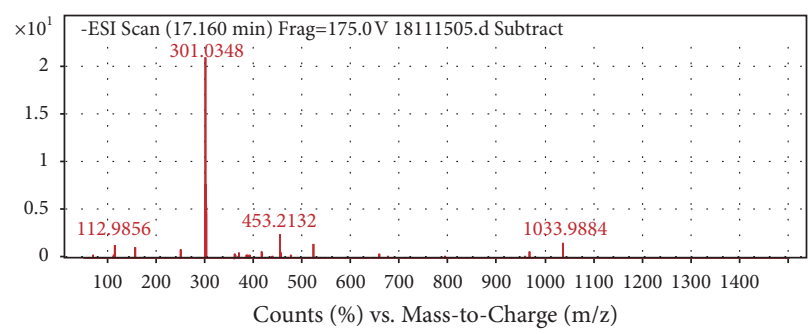

(e)

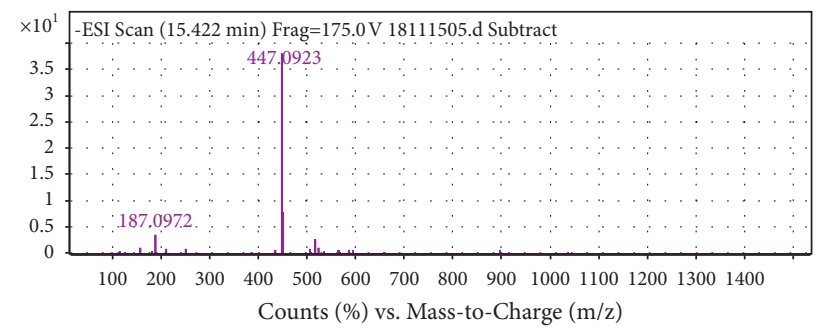

(d)

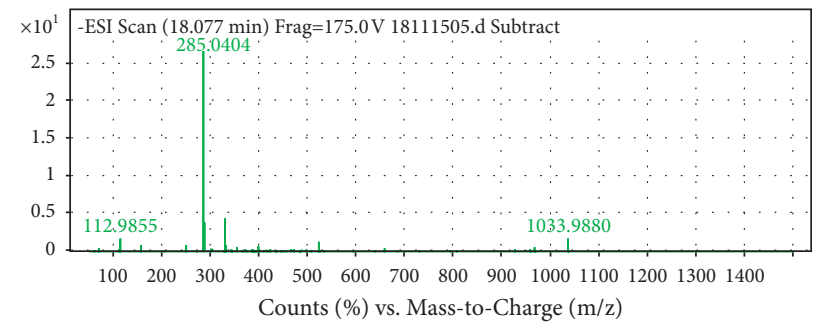

(f)

Figure 7: Mass chromatogram of components in EAE $(4 \mathrm{mg} / \mathrm{mL})$. (a) Mass chromatogram of rutin. (b) Mass chromatogram of isoquercetin. (c) Mass chromatogram of kaempferol-3-o-rutoside. (d) Mass chromatogram of astragaloside. (e) Mass chromatogram of quercetin. (f) Mass chromatogram of kaempferol.

TABLE 1: Molecular weight and formula of six chemical components in EAE $(4 \mathrm{mg} / \mathrm{mL})$.

\begin{tabular}{lccccc}
\hline No. & $\mathrm{tR} / \mathrm{min}$ & Molecular formula & Measured value $(\mathrm{m} / z)$ & Theoretical value $(\mathrm{m} / z)$ & Relative error $(\mathrm{ppm})$ \\
\hline 1 & 14.393 & $\mathrm{C}_{27} \mathrm{H}_{30} \mathrm{O}_{16}$ & 610.1494 & 610.1473 & 2.31 \\
2 & 14.682 & $\mathrm{C}_{21} \mathrm{H}_{19} \mathrm{O}_{12}$ & 464.0955 & 464.0944 & 2.24 \\
3 & 15.181 & $\mathrm{C}_{27} \mathrm{H}_{29} \mathrm{O}_{15}$ & 594.1585 & 594.1576 & 1.41 \\
4 & 15.422 & $\mathrm{C}_{21} \mathrm{H}_{19} \mathrm{O}_{11}$ & 448.1006 & 448.0996 & 2.19 \\
5 & 17.160 & $\mathrm{C}_{15} \mathrm{H}_{9} \mathrm{O}_{7}$ & 302.0427 & 302.0421 & 1.82 \\
6 & 18.077 & $\mathrm{C}_{15} \mathrm{H}_{9} \mathrm{O}_{6}$ & 286.0477 & 286.0477 & 0.09 \\
\hline
\end{tabular}

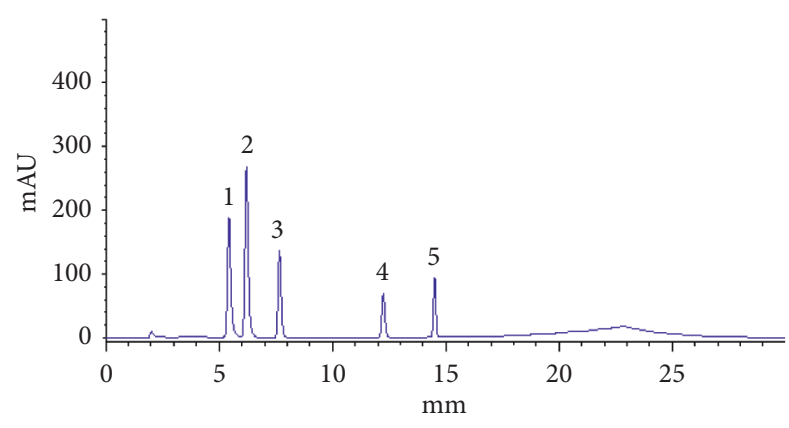

(a)

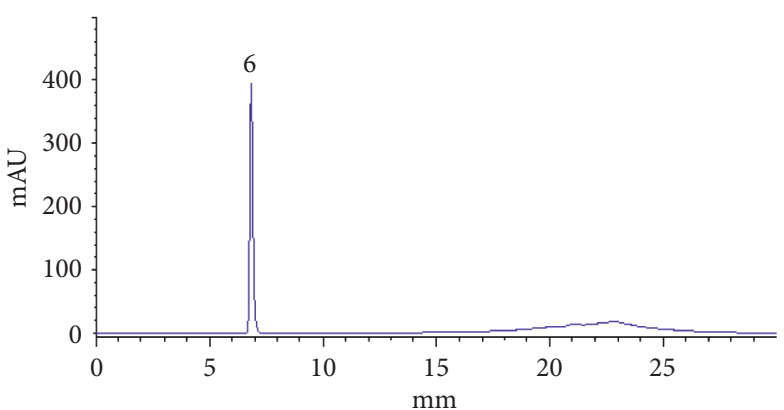

(b)

FIgURE 8: Continued. 


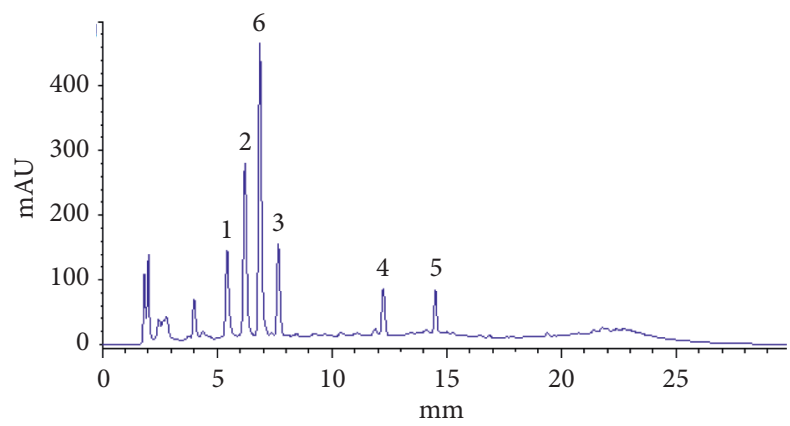

(c)

Figure 8: Chromatogram of standard reference and EAE $(4 \mathrm{mg} / \mathrm{mL})$. (a) Chromatogram of mixed standard reference. (b) Chromatogram of kaempferol-3-o-rutoside standard reference. (c) Chromatogram of EAE: 1, rutin; 2, isoquercetin; 3, astragaloside; 4, quercetin; 5, kaempferol; 6, kaempferol-3-o-rutoside.

dysfunction in male mice by increasing the antioxidant enzyme activities and inhibiting inflammation [63, 64]. Kaempferol and kaempferol-3-o-rutoside increases the $\mathrm{DPPH}$ and ABTS free radical scavenging activity and inhibits the T cell proliferation induced by concanavalin $\mathrm{A}$ and nitric oxide (NO) or reactive oxygen species (ROS) production in LPS-induced 264.7 macrophages cells [65].

\section{Conclusions}

In this study, for the first time, we used in vitro traditional antioxidant tests (DPPH assay, TEAC assay, FRAP, and hydroxyl radical scavenging assay) combined with HPLCDPPH and HPLC-hydroxyl radical scavenging methods to screen out EAE with high antioxidant activity from Tetrastigma hemsleyanum and identified six antioxidant components by Q-TOF LC/MS and HPLC. The EAE may be used in food, medicine, and other fields to prevent the disease and assist the treatment of some oxidative stress-related diseases as these six compounds have significant biological activities. However, further studies are needed on the correlation between the content of each antioxidant component in EAE and the antioxidant capacity of EAE and the antioxidant mechanism.

\section{Abbreviations}

DPPH:

1,1-Diphenyl-2-picrylhydrazyl radical, 2,2-diphenyl-1-(2,4,6-trinitrophenyl) hydrazyl

TEAC: $\quad$ Trolox equivalent antioxidant capacity

FRAP: $\quad$ Ferric reducing antioxidant power

ABTS: $\quad$ 2, 2'-Azino-bis(3-ethylbenzothiazoline-6sulfonic acid)

HPLC: $\quad$ High-performance liquid chromatography

HPLC-Q-TOF High-performance liquid

LC/MS: chromatography coupled with quadrupole time-of-flight tandem mass spectrometry

EAE: $\quad$ The ethyl acetate extract

CE: $\quad$ The crude extract
PEE: $\quad$ Petroleum ether extract

BE: $\quad$ n-Butanol extract

BHT: $\quad$ Dibutyl hydroxytoluene

BHA: Butyl hydroxyanisole

PG: $\quad$ Propyl gallate

TPTZ: 2,4,6-Tri-2-pyridinyl-1,3,5-triazine

Trolox: 6-Hydroxy-2,5,7,8-tetramethylchroman2-carboxylic acid.

\section{Data Availability}

The datasets generated and analyzed during the present study are available from the corresponding author upon request.

\section{Conflicts of Interest}

The authors declare that they have no conflicts of interest.

\section{Acknowledgments}

This work was supported by the Ningbo Natural Science Foundation (2018A610244) and the science and technology benefiting people technology R\&D project of Ningbo Science and Technology Bureau (2015C50063).

\section{References}

[1] S. B. Liao, W. W. Cai, D. Chen, P. Xie, J. Huang, and Z. X. Mu, "Anti-inflammatory and analgesic effects of the extracts of Tetrastigmais hemsleyanum's aerial parts form Fujian in vivo," Chinese Journal of Modern Applied Pharmacy, vol. 34, no. 2, pp. 319-324, 2017.

[2] W. Dan-Dan, G. Rong, and Y. Bin, "Experimental study on the effects of Radix Tetrastigmae on respiratory syncytial virus," Natural Product Research and Development, vol. 31, pp. 1070-1074, 2019.

[3] X. M. Zhong, Q. Q. Mao, and Z. Huang, "Experimental study on the protective effect and acute toxicity of Tetrastigma hemsleyanum extract on $\mathrm{CCl} 4$ induced acute liver injury in mice," Chinese Traditional Patent Medicine, vol. 28, no. 3, pp. 422-424, 2006. 
[4] L. I. Ping, J. I. Weiwei, P. Xin, and D. O. Pathology, "Study on the trifolium extract in anit-induced liver injury in mice," China Modern Doctor, vol. 56, no. 30, pp. 32-35, 2018.

[5] H. Y. Wu, Y. T. Wu, Y. Y. Chen, H. L. Luo, and H. F. Jiang, "Effect of Radix Tetrastigma hemsleyani flavones on apoptosis and MAPK signaling pathway in myeloid leukemia NB-4 cells," Chinese Journal of Pathophysiology, vol. 35, no. 8, pp. 1451-1456, 2019.

[6] T. Hu, Z. Q. Feng, and L. R. Zhong, "Effect of flavonoids from Tetrastigma hemsleyani Diels et Gilg on myeloid derived suppressive cells in mice bearing Lewis lung cancer," Chinese Journal of Integrated Traditional and Western Medicine, vol. 38, no. 10, pp. 1229-1233, 2018.

[7] J. N. M. College, Dictionary of Traditional Chinese Medicine, Shanghai People's Publishing House, Shanghai, China, 1977.

[8] X. L. Wang, "Influence of the water extract of Radix Tetrastigmae on IL-23 and IL-17 in rats of rats of chronic obstructive pulmonary disease," Journal of Practical Traditional Chinese Medicine, vol. 32, no. 9, pp. 848-850, 2016.

[9] D. D. Liu, G. Cao, Q. Zhang, Y. L. Ye, L. K. Han, and W. H. Ge, "Inhibition effect of flavonoids from Radix tetrastigmae on acute lung injury of aged mice through p38MAPK and NF- $\kappa \mathrm{B}$ pathway," Chinese Pharmacological Bulletin, vol. 12, pp. 1725-1729, 2015.

[10] D. Liu, J. H. Ju, and J. S. Yang, "Studies on chemical sonstituents form Tetrastigma hypoglaucum," Chinese Journal of traditional Chinese Medicine, vol. 34, no. 1, pp. 4-6, 2004.

[11] D. Liu and J. S. Yang, "A Study on chemical components of Tetrastigma hypoglaucum Diels et Gilg. native to China," Chinese Traditional and Herbal Drugs, vol. 24, no. 10, pp. 611-612, 1999.

[12] Y. J. Hu, L. Cheng, and J. B. Pu, "GC-MS analysis of petroleum ether extract from Testrastigma hemsleyanum," Chinese Traditional Medicine Science and Technology, vol. 20, no. 1, pp. 46-47, 2013.

[13] Y. Q. Li, W. C. Lu, and Y. Z. Guo, "Studies on the chemical constituents of Testrastigma hemsleyanum Diels et Gilg," Chinese Traditional and Herbal Drugs, vol. 34, no. 11, pp. 982-983, 2003.

[14] F. Shi-Ming, X. Xin-Yue, Z. Fan-Tian, Z. Xiao-Hong, C. BiYa, and X. U. Wen, "Identification of chemical components and determination of flavonoids in Tetrastigma hemsleyanum leaves," Chinese Journal of Pharmaceutical Analysis, vol. 37, no. 8, pp. 1481-1486, 2017.

[15] M. L. Zeng, N. T. Shen, S. W. Wu, and Q. Li, "Analysis on chemical constituents in Tetrastigma hemsleyanum by UPLCTriple-TOF/MS," Chinese Traditional and Herbal Drugs, vol. 4, no. 5, pp. 874-883, 2017.

[16] Y. Jiang, Q. Zheng, A. Zhang, L. Cui, L. Xia, and M. Luo, "Flavone from Zhongjiefeng (Herba Sarcandrae Glabrae) inhibits platelet apoptosis in immune-induced bone marrow failure through mitochondrial pathway," Journal of traditional Chinese medicine $=$ Chung $i$ tsa chih ying wen pan, vol. 37, no. 5, pp. 643-649, 2017.

[17] C. L. Peng, S. M. Zhang, and Y. Zhu, "Differential inhibition effects of gingkgo flavones and ginkgolide on the proliferation of vascular smooth muscle cell and the mechanism," Central South Pharmacy, vol. 15, no. 10, pp. 1351-1356, 2017.

[18] L. Lu, J. Mi, and Q. Luo, "Optimization of extraction process of flavonoids from lycium barbarum L. var. Auranticarpum K. F. Ching and its antioxidant activities in vitro," Science and Technology of Food Industry, vol. 40, no. 24, pp. 165-171, 2019.
[19] W. Yan-Bo, S. Yan, and Y. Yi-Jun, "Ultrasonic assisted extraction of polyphenols and flavonoids from Maijishan wild acanthopanax and their antioxidant activities in vitro," Natural Product Research and Development, vol. 31, no. 12, pp. 2153-2162, 2019.

[20] J. J. Diao, R. A. Cao, and C. Y. Li, "Antioxidant activity and structure of flavonoid compounds extracted from raspberry root," Chinese Journal of Biologicals, vol. 32, no. 12, pp. 1350-1357, 2019.

[21] H. Jin, J. Yu, and Z. Y. Wang, "Mechanism of flavonoids of sophorae fructus in inhibiting proliferation, migration and invasion of hepatocellular carcinoma cells by regulating LncRNA FBXL 19-AS1/miR-342-3p pathway," China Journal of Chinese Materia Medica, vol. 10, 2020.

[22] Y. S. Qi, X. Li, and X. M. Qin, "Anti- cancer effect of the flavonoids of Astragalus combined with cisplatin on lewis lung carcinoma-bearing mice," Acta Pharmaceutica Sinica, vol. $8,2020$.

[23] L. H. Zhang, T. T. Wu, L. G. Zhao, X. Wei, S. H. Zhang, and C. X. Wang, "Advances in studies anticancer activity of flavonoids from Ginkgo biloba extract," Chinese Pharmaceutical Journal, vol. 54, no. 6, pp. 444-449, 2019.

[24] G. G. Lv, Z. K. Qiu, S. Z. Chang, H. Lin, J. Lu, and D. D. Ye, "Anti-inflammatory, analgesic and analgesic effects of total flavone from Stigma maydis and its effect on acute gouty arthritis," Drug Evaluation Research, vol. 41, no. 2, pp. 206209, 2018.

[25] A. S. Mecenas, C. R. Adão Malafaia, L. S. Sangenito et al., "Rutin derivatives obtained by transesterification reactions catalyzed by Novozym 435: antioxidant properties and absence of toxicity in mammalian cells," PLoS One, vol. 13, no. 9, Article ID e0203159, 2018.

[26] L. Živković, V. Bajić, M. Bruić et al., "Antigenotoxic and antioxidant potential of medicinal mushrooms (Immune Assist) against DNA damage induced by free radicals-an in vitro study," Mutation Research, vol. 845, Article ID 403078, 2019.

[27] R. Rohman and R. Kar, "How does the presence of an oxyradical influence the behavior of polyphenolic antioxidant? A case study on gallic acid," Journal of Molecular Modeling, vol. 24, no. 7, p. 165, 2018.

[28] Y. Sun, K. Yang, Q. Cao et al., "Homogenate-assisted vacuumpowered bubble extraction of moso bamboo flavonoids for on-line scavenging free radical capacity analysis," Molecules, vol. 22, no. 7, 2017.

[29] P. Xiao, H. Huang, X. Li, J. Chen, and J.-a. Duan, "Characterization, evaluation of nutritional parameters of Radix isatidis protein and its antioxidant activity in D-galactose induced ageing mice," BMC Complementary and Alternative Medicine, vol. 19, no. 1, p. 297, 2019.

[30] M. D. Mira-Sánchez, J. Castillo-Sánchez, and J. M. MorillasRuiz, "Comparative study of rosemary extracts and several synthetic and natural food antioxidants. Relevance of carnosic acid/carnosol ratio," Food Chemistry, vol. 309, Article ID 125688, 2020.

[31] A. M. Mahmoud, F. L. Wilkinson, M. A. Sandhu, J. M. Dos Santos, and M. Y. Alexander, "Modulating oxidative stress in drug-induced injury and metabolic disorders: the role of natural and synthetic antioxidants," Oxidative Medicine and Cellular Longevity, vol. 2019, Article ID 3206401, 5 pages, 2019.

[32] I.-C. Sheih, T.-K. Wu, and T. J. Fang, "Antioxidant properties of a new antioxidative peptide from algae protein waste 
hydrolysate in different oxidation systems," Bioresource Technology, vol. 100, no. 13, pp. 3419-3425, 2009.

[33] N. Chen, H. Yang, Y. Sun, J. Niu, and S. Liu, "Purification and identification of antioxidant peptides from walnut (Juglans regia L.) protein hydrolysates," Peptides, vol. 38, no. 2, pp. 344-349, 2012.

[34] B. L. Zhao, Oxygen Free Radicals and Natural Antioxidants, Science Press, Beijing, China, 1999.

[35] Z. Li, H. Caiyun, and R. Fazheng, "Anti-obesity efficacy of extracts from several kinds of tea in high-fat diet-induced obese mice," Food Science, vol. 39, no. 17, pp. 192-198, 2018.

[36] L. I. Yan-Hui, L. Miao-Ling, L. Si-Hui, S. U. Han-Tao, and S. Hai-Yan, "Effects of tea extracts of different fermentations on proliferation of colorectal cancer cell line HCT116," Guangdong Agricultural Sciences, vol. 45, no. 9, pp. 103-108, 2018.

[37] L. Liu, L. Yu, and M. Zhou, "Intervention study on that effect of grape seed extract proanthocyanidins on blood glucose and blood lipid in the patients with type 2 diabetes mellitus," International Journal of Laboratory Medicine, vol. 40, no. 15, pp. 1844-1849, 2019.

[38] L. Zhang and S. Lei, "Protective effects and mechanism of Momordica Charantia extract on RGCS of type 2 diabetic rats," Chinese Journal of Coal Industry Medicine, vol. 23, no. 1, pp. 11-15, 2020.

[39] H. Wang, Q. Chi, S. L. Xiong, C. F. Zeng, and L. Wan, "Effects of Lonicera japonica extract on lung inflammation in LPSinduced ARDS rats," Journal of Guangdong Pharmaceutical University, vol. 33, no. 3, pp. 379-382, 2017.

[40] X. Zhang, M. Y. Shi, M. Sun, Y. B. Zhang, Y. L. Zhang, and L. J. Liu, "Influences of Shengdihuang(Rehmanniae recens Radix) extract on pulmonary index and monocyte chemoattractant protein-1 mRNA expression of pulmonary interstitial fibrosis rats," LiShiZhen Medicine and Materia Medica Research, vol. 29, no. 12, pp. 2884-2886, 2018.

[41] X. Y. Tang and Y. L. Zhao, "Study on the effect of garlic extracts on preservation and antioxidant activity of chilled meat," Meat Industry, vol. 37, no. 7, pp. 435-438, 2017.

[42] N. Huda-Faujan, A. Noriham, A. S. Norrakiah, and A. S. Babji, "Antioxidant activity of plants methanolic extracts containing phenolic compounds," African Journal of Biotechnology, vol. 8, 2009.

[43] W. Khan, S. Subhan, D. F. Shams et al., "Antioxidant potential, phytochemicals composition, and metal contents of Datura alba," BioMed Research International, vol. 2019, Article ID 2403718, 8 pages, 2019.

[44] L. I. Meng-Ru, Z. Yu-Zhi, D. U. Guan-Hua, and Q. Xue-Mei, "Research progress about the anti-aging effect and mechanism of flavonoids from traditional Chinese medicine," Acta Pharmaceutica Sinica, vol. 54, no. 8, pp. 1382-1391, 2019.

[45] G. L. Wang, J. K. Li, X. Wu, and K. Wang, "Extraction and purification of Debregeasia orientalis fruit polyphenols and its antioxidant, antitumor activity," Natural Product Research and Development, vol. 31, no. 1, pp. 1-9, 2019.

[46] L. Ding and J. Q. Xiong, "Study preliminary on the anti-tumor effect of the extract of Tetrastigmatis hemsleyani," Strait Pharmaceutical Journal, vol. 23, no. 12, pp. 46-48, 2011.

[47] L. Ding, L. X. Zhang, Y. Qiu, and Y. H. Wang, "Chemical constituents in chloroform extraction of Tetrastigmatis hemsleyani Diels et. Gilg and their antitumor activities," Chinese Pharmaceutical Journal, vol. 50, no. 21, pp. 18571860, 2016.

[48] L. Bao, X. F. Ma, and X. H. Song, "Two new resveratrol tetramers isolated from Cayratia japonica (thunb.) gagn. With strong inhibitory activity on fatty acid synthase and antioxidant activity," Chemistry and Biodiversity, vol. 7, no. 12, pp. 2931-2940, 2011.

[49] S. Yong, Chemical composition, activities of anti-oxidation and anticancer of Terastigma hemsleyanum, JiangXi: NanChang University, Nanchang, China, 2018.

[50] G. Shui, "An investigation of antioxidant capacity of fruits in Singapore markets," Food Chemistry, vol. 76, no. 60-75, 2002.

[51] P. Stratil, B. Klejdus, and V. Kubáň, "Determination of total content of phenolic compounds and their antioxidant activity in vegetables evaluation of spectrophotometric methods," Journal of Agricultural and Food Chemistry, vol. 54, no. 3, pp. 607-616, 2006.

[52] I. F. F. Benzie and J. J. Strain, "The ferric reducing ability of plasma (FRAP) as a measure of "antioxidant power": the FRAP assay," Analytical Biochemistry, vol. 239, no. 1, pp. 70-76, 1996.

[53] M. Jin, Y. X. Cai, J. R. Li, and H. Zhao, “1, 10-Phenanthroline$\mathrm{Fe} 2+$ oxidative assay of hydroxyl radical produced by $\mathrm{H} 2 \mathrm{O} 2 /$ Fe2+," Progress in Biochemistry and Biophysics, vol. 23, no. 6, pp. 553-555, 1996.

[54] J. D. Fu, New Method for Rapid Screening of Antioxidants from Nature Products, pp. 14-54, ZheJiang University, ZheJiang, China, 2010.

[55] W. M. Brand-Williams, M. E. Cuvelier, and C. L. W. T. Berset, "Use of a free radical method to evaluate antioxidant activity," Lebensmittel-Wissenschaft und-Technologie-Food Science and Technology, vol. 28, no. 1, 1995.

[56] X. Duan, Y. Jiang, X. Su, Z. Zhang, and J. Shi, “Antioxidant properties of anthocyanins extracted from litchi (Litchi chinenesis Sonn.) fruit pericarp tissues in relation to their role in the pericarp browning," Food Chemistry, vol. 101, no. 4, pp. 1365-1371, 2007.

[57] H. T. H. Vu, S. M. Hook, S. D. Siqueira, M. Anette, R. Thomas, and M. D. Arlene, "Are phytosomes a superior nanodelivery system for the antioxidant rutin?” International Journal of Pharmaceutics, vol. 548, 2018.

[58] Y. W. Han, C. C. Wang, and L. X. Ming, "Protective effect of rutin on early brain injury in mice with subarachnoid hemorrhage by anti-oxidation and anti-apoptosis," Drugs \& Clinic, vol. 35, no. 3, pp. 421-425, 2020.

[59] W. Xu, Z. Q. Fu, J. Lin, X. C. Huang, and S. M. Fan, "Qualitative and quantitative analysis of major constituents in Tetrastigma hemsleyanum by HPLC-Q-TOF-MS and UPLCQqQ-MS," Zhongguo Zhong yao za zhi = Zhongguo zhongyao zazhi = China journal of Chinese materia medica, vol. 39, no. 22, pp. 4365-4372, 2014.

[60] X. U. Wen, F. U. Zhi-Qin, L. Jing, H. Xue-Cheng, Y. U. HongMin, and H. Ze-Hao, "Rapid simultaneous determination of ten major flavonoids in Tetrastigma hemsleyanum by UPLCMS/MS," Yao xue xue bao = Acta pharmaceutica Sinica, vol. 49, no. 12, pp. 1711-1717, 2014.

[61] A. O. Ademosun, G. Oboh, F. Bello, and P. O. Ayeni, "Antioxidative properties and effect of quercetin and its glycosylated form (rutin) on acetylcholinesterase and butyrylcholinesterase activities," Journal of Evidence-Based Complementary and Alternative Medicine, vol. 21, 2016.

[62] C.-P. Wang, Y.-W. Shi, M. Tang et al., "Isoquercetin ameliorates cerebral impairment in focal ischemia through antioxidative, anti-inflammatory, and anti-apoptotic effects in primary culture of rat hippocampal neurons and hippocampal CA1 region of rats," Molecular Neurobiology, vol. 54, no. 3, pp. 2126-2142, 2017. 
[63] B. H. Xia, Y. M. Zhou, S. L. Pi, and e al, "Determination of antioxidant activity of isoquercetin, rutin and astragaloside in mulberry leaves by UPLC," Journal of Chinese Medicinal Materials, vol. 39, no. 3, pp. 586-589, 2016.

[64] S. O. Abarikwu, A. B. Pant, and E. O. Farombi, "Dietary antioxidant, quercetin, protects sertoli-germ cell coculture from atrazine-induced oxidative damage," Journal of Biochemical and Molecular Toxicology, vol. 26, no. 11, pp. 477485, 2012.

[65] W. Jingqiu, F. Xianying, G. Lin, C. Fuliang, Z. Linguo, and W. Zhenzhong, "Antitumor, antioxidant and anti-inflammatory activities of kaempferol and its corresponding glycosides and the enzymatic preparation of kaempferol," PLoS One, vol. 13, no. 5, Article ID e0197563, 2018. 